\title{
Crioterapia prostática como tratamiento primario en pacientes con cáncer de próstata
}

\author{
Bjerklund Johansen TE. \\ Urology Section, Telemark Hospital, Porsgrunn, Norway. \\ Actas Urol Esp. 2007;31(6):651-659
}

\begin{abstract}
RESUMEN
CRIOTERAPIA PROSTÁTICA COMO TRATAMIENTO PRIMARIO EN PACIENTES CON CÁNCER DE PRÓSTATA

Objetivos: La ablación crioterápica prostática (CSA) es una de las opciones de tratamiento que se han desarrollado, siendo considerada, actualmente, como una alternativa terapéutica en la enfermedad localizada. El objetivo de este trabajo es presentar la experiencia preliminar con CSA en cáncer de próstata en un centro de Noruega.

Material y Métodos. Un total de 132 pacientes con cáncer de próstata han sido tratados con CSA desde Septiembre de 2003. En 90 pacientes, la CSA fue utilizada como tratamiento primario. Estos pacientes presentaban enfermedad de bajo riesgo LR ( $\mathrm{n}=27$ ), riesgo intermedio IR $(\mathrm{n}=37)$ y alto riesgo HR $(\mathrm{n}=26)$. Los pacientes de bajo riesgo se definieron con las siguientes características: T1a-T2a, NO, MO, una puntuación de Gleason $\leq 6$ y antígeno prostático específico de $(\mathrm{PSA})<10 \mathrm{ng} / \mathrm{ml}$. Pacientes de riesgo intermedio eran aquellos con tumores T2b o un PSA entre $10-20 \mathrm{ng} / \mathrm{ml}$ o una puntuación de Gleason de 7 . Los pacientes de alto riesgo se definieron como aquellos tumores T2 o con una puntuación de Gleason $>7$ o un PSA $>20 \mathrm{ng} / \mathrm{ml}$. Los restantes 42 pacientes tenían enfermedad localmente avanzada o habían recibido tratamiento de rescate después de radioterapia externa (RTE), crioterapia o tratamiento hormonal. Los pacientes fueron evaluados a 3 y 6 meses y posteriormente a intervalos de 6 meses. Se realizó una historia funcional mediante flujometria y volumen residual.

Resultados: Los resultados se presentan en aquellos pacientes que han recibido CSA como tratamiento primario, el seguimiento más largo es 42 meses. La mediana de seguimiento es de 21 meses y un $39 \%$ de los pacientes han sido vistos en la revisión de los 24 meses. No hay evidencia de progresión clínica en los pacientes en el grupo de bajo y alto riesgo (LH-RH). En el grupo de riego intermedio (IR) 2 pacientes evidenciaron progresión clínica. Los efectos secundarios más comunes durante el primer año post CSA fueron la obstrucción del tracto urinario y la necesidad de extirpar el tejido prostático necrosado o calcificado. El análisis histopatológico del tejido extraído no evidenció la presencia de cáncer de próstata. Todos los pacientes en los grupos de riesgo bajo e intermedio que eran sexualmente activos antes del tratamiento presentaban buena actividad sexual en el último seguimiento, 37\% utilizaba algún tipo de ayuda. $86 \%$ de los pacientes de los grupos de riesgo bajo e intermedio no presentaron molestias en la esfera sexual.

Conclusiones: La CSA está reconocida por la EAU, actualmente, como una alternativa terapéutica verdadera para el tratamiento del cáncer de próstata. Muchas instituciones ofrecen CSA sólo a pacientes ancianos debido a la ausencia de resultados a largo plazo. Si los resultados a largo plazo confirman los resultados obtenidos a corto plazo por mi serie, y si otras series obtienen efectos secundarios comparables, CSA podría ser ofrecido como tratamiento primario a pacientes jóvenes.
\end{abstract}

Palabras Clave: Crioterapia. Cáncer de próstata. Progresión PSA. Progresión clínica. Efectos secundarios. Terapias mínimamente invasivas.

\section{ABSTRACT}

CRYOSURGICAL ABLATION AS PRIMARY TREATMENT IN PROSTATE CANCER PATIENTS

Objective: Cryosurgical ablation (CSA) is one treatment option that has been developed and it is now recognised as a true therapeutic alternative in the treatment of localised disease. The aim of this paper is to present the preliminary experience with CSA in prostate cancer from the centre in Norway.

Materials and methods: A total of 132 patients with prostate cancer have been treated with CSA since September 2003. In 90 patients, CSA was the primary therapy used and these patients included those with low (LR; $n=27$ ), intermediate (IR; $n=37$ ) and high risk (HR; $\mathrm{n}=26$ ) disease. Low risk patients were defined as those with T1a-T2a, NO, MO, Gleason score $\leq 6$ and prostate-specific antigen (PSA) $<10$ $\mathrm{ng} / \mathrm{ml}$. Intermediate risk were patients with T2b tumours or a PSA level of $10-20 \mathrm{ng} / \mathrm{ml}$ or Gleason score 7 . High risk were defined as those with T2c tumours or Gleason score $>7$ or PSA $>20 \mathrm{ng} / \mathrm{ml}$. The remaining 42 patients either had locally advanced disease or had received salvage treatment after previous external beam radiation (EBRT), cryotherapy or hormone treatment. Patients were evaluated at 3 and 6 months and thereafter at 6 month intervals. A voiding history was taken and the following tests conducted: uroflowmetry, residual urine.

Results: Results are presented for those patients receiving CSA as a primary therapy. Currently, the longest follow-up period is 42 months. The median observation period is 21 months and $39 \%$ of patients have been seen at the 24-month follow-up visit. No evidence of clinical progression has been observed in patients in the LR or HR group. Two patients in the IR group had clinical evidence of progression. The most common side effects seen during the first year post CSA were urinary tract obstruction and the need for removal of dead prostatic tissue or calcifications. Histological examination of the removed tissue did not shown evidence of prostate cancer. All patients in the LR and IR group that were sexually active before treatment were also sexually active on last follow-up, $37 \%$ using some kind of aid. $86 \%$ of patients in the LR and IR groups reported no bother as to sexual function.

Conclusions: CSA is now recognised by the EAU as a true therapeutic alternative for the treatment of prostate cancer. Many institutions offer CSA only to older patients due to the lack of long-term data. If longer follow-up data confirm the short-term effect seen in my series, and if comparable side effects are reported in other studies, CSA might also be offered as primary treatment to younger patients.

Keywords: Cryoablation. Prostate cancer. PSA progression. Clinical progression. Side-effects. Minimally invasive therapy. 
$\mathrm{L}$ a regla de oro para el tratamiento quirúrgico del cáncer de próstata es la Prostatectomía radical. A pesar de esto, el único estudio aleatorizado comparando prostatectomía radical con tratamientos conservadores no demostró mejoría en la supervivencia a 5 años $^{1}$. Se deben considerar, también, los efectos secundarios de la prostatectomía radical, siendo la incontinencia urinaria y la disfunción eréctil los más importantes ${ }^{2}$. Además, hay que añadir la pérdida sanguínea durante el procedimiento, cuya media es $1 \mathrm{dm}^{3}$ (3). Los datos de los pacientes noruegos con cáncer de próstata tratados con prostatectomía radical indican un porcentaje de márgenes positivos que varía entre 15 y 50\% ${ }^{4,5}$. Las terapias mínimamente invasivas para el cáncer de próstata localizado han sido el objetivo objeto de múltiples investigaciones durante los últimos años con el objetivo de reducir los efectos secundarios observados con la Prostatectomía radical. La ablación por crioterapia (CSA) es uno de las opciones terapéuticas que se han desarrollado y ahora es reconocida como una verdadera alternativa en el tratamiento de la enfermedad localizada ${ }^{3,6}$. El objetivo de este trabajo es presentar la experiencia preliminar en el tratamiento del cáncer de próstata con ablación por crioterapia (CSA) en un centro de Noruega.

\section{MATERIAL Y MÉTODOS \\ Pacientes}

Un total de 132 pacientes con cáncer de próstata fueron tratados con CSA desde Septiembre de 2003. En 90 pacientes, se utilizó la CSA como tratamiento primario. Estos pacientes eran de riesgo bajo (LR, $n=27$ ), riesgo intermedio (IR, $\mathrm{n}=37)$ y alto riesgo ( $\mathrm{HR}, \mathrm{n}=26)$. Los pacientes de bajo riesgo se definieron con las siguientes características: T1a-T2a, NO, MO, una puntuación de Gleason $\leq 6 \mathrm{y}$ antígeno prostático específico de (PSA) $<10 \mathrm{ng} / \mathrm{ml}$. Pacientes de riesgo intermedio eran aquellos con tumores T2b o un PSA entre 10-20 ng/ml o una puntuación de Gleason de 7 . Los pacientes de alto riesgo se definieron como aquellos tumores T2c o con una puntuación de Gleason $>7$ o un PSA $>20 \mathrm{ng} / \mathrm{ml}$. Los restantes 42 pacientes tenian enfermedad localmente avanzada o habían recibido tratamiento de rescate después de radioterapia externa (RTE), crioterapia o tratamiento hormonal.

\section{Técnica}

EL procedimiento se realizó utilizando una máquina Galil/Oncura cryosystem de $3^{\text {a }}$ generación con unas agujas y termosensores de 17G Seed Net. El equipo de ecografia fue un B\&K Falkon 2101 con una sonda 8658 (B6K Dinamarca) un braquistand con pedal y un braqui standoff en la sonda configuró el resto del equipo (Fig. 1). El braqui standoff era una sábana de plástico colocada alrededor de la sonda con una ventana para la expansión del colector interno cuando se inflaba con agua. Para congelar se utilizó Gas Argón y Gas Helio para el calentamiento.

Se entregó información por escrito del procedimiento a los pacientes y todos ellos dieron su consentimiento, después de haber sido informados sobre otras posibilidades terapéuticas y los efectos secundarios esperados de la criocirugia. La mayoría de los procedimientos se realizaron bajo anestesia epidural o anestesia general para asegurarse que el paciente no se movía. El procedimiento de CSA, incluyendo la inserción de las

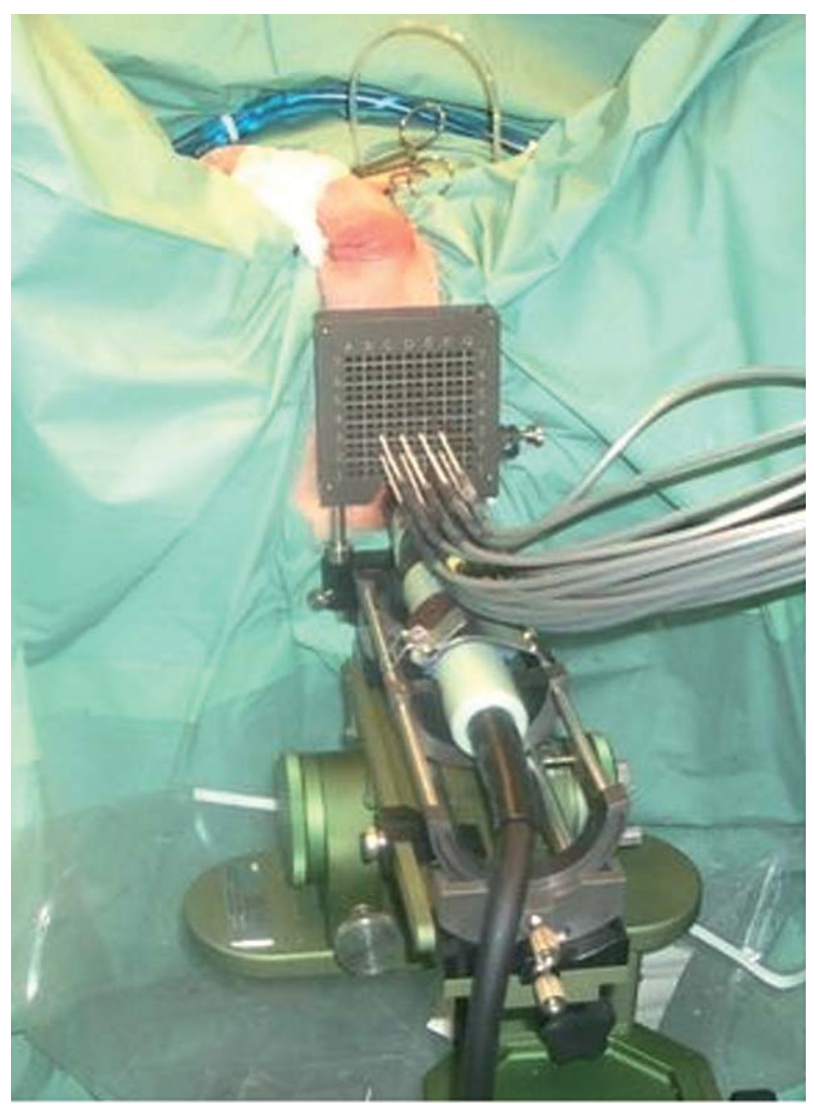

FIGURA 1. Braquistand con sonda ecográfica y las agujas insertadas en la próstata. 
agujas y de los periodos de congelación y calentamiento, fue realizado como se ha descrito previamente y de acuerdo con las guías clínicas de la Asociación Europea de Urología (EAU) de cáncer de próstata ${ }^{8}$, (Figs. 2 y 3).

El catéter supra-púbico se clampa, entre los días 1 y 2 del post-operatorio y se retira después de comprobar que el paciente es capaz de vaciar su vejiga, antes del alta. De otra manera el paciente es dado de alta con el catéter y éste se retira cuando el paciente orina de una manera aceptable.

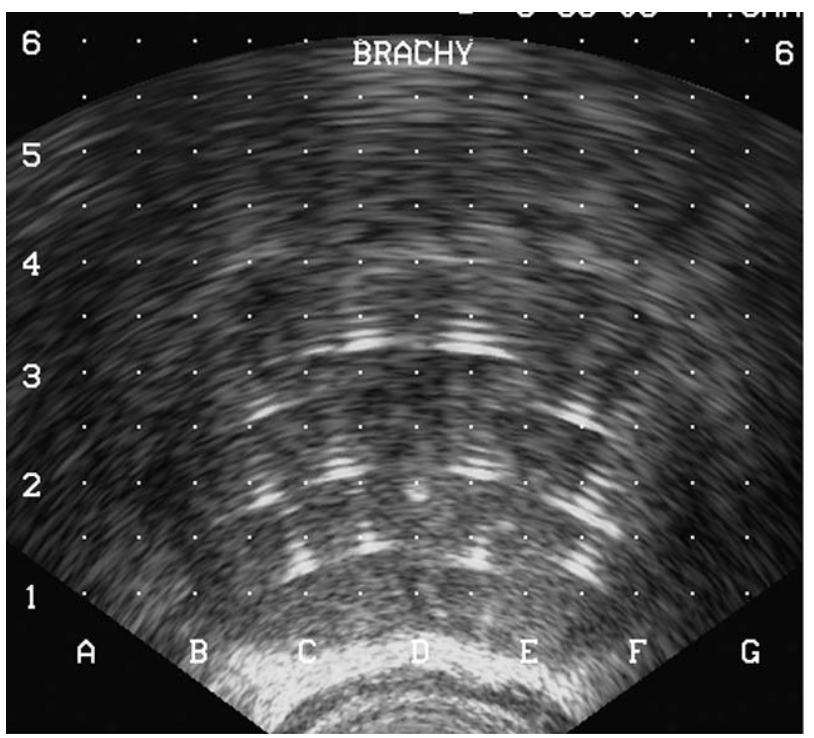

FIGURA 2. Imagen ecográfica de un corte transversal de la próstata que muestra la red de agujas insertadas en la próstata y la guía en la uretra.

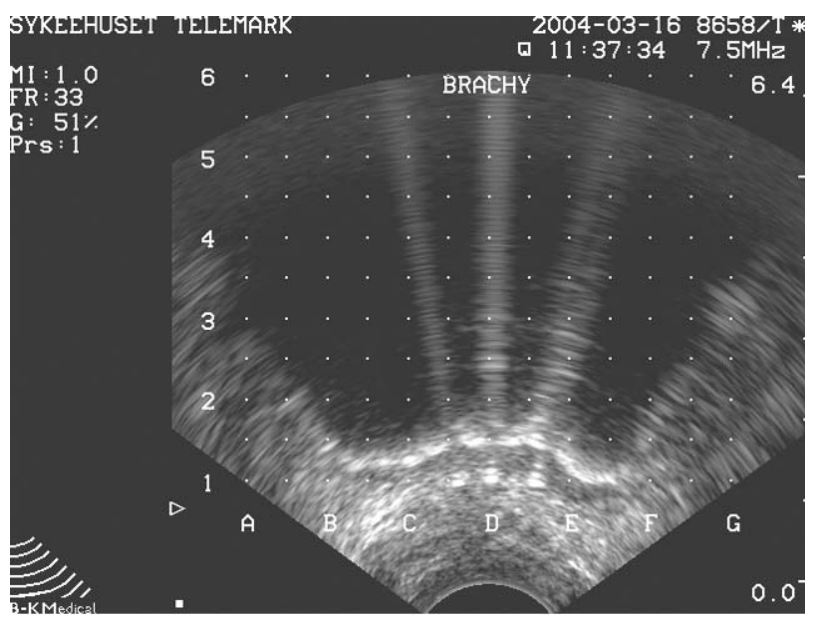

FIGURA 3. Imagen ecográfica de un corte transversal de la próstata que muestra la bola de hielo expandiéndose posteriormente hacia el termosensor en el centro y las agujas de calentamiento en ambos lados.

\section{Seguimiento}

Los pacientes fueron evaluados a los meses 3 y 6 y después a intervalos de 6 meses. Se realizó una historia de su hábito miccional así como las siguientes pruebas: flujometría, volumen residual, PSA y tacto rectal (DRE). En función de la evolución del PSA así como de la evolución clínica se indicó la realización de una biopsia prostática. Los pacientes eran preguntados por su actividad sexual y sus alteraciones. La actividad sexual se clasificó en activo sin ayuda, activo con la utilización de ayuda, o inactivo. La actividad sexual se clasificó en sin alteración, con alguna alteración, o con alteración significativa.

La progresión clínica se determinó en función del diagnóstico de metástasis a distancia en pruebas de imagen y en una biopsia de próstata positiva y en el inicio de tratamiento hormonal.

La progresión de PSA se definió como un ascenso del PSA por encima del punto de corte recomendado por la Sociedad Americana de Oncologia Radioterápica (ASTRO) ${ }^{9}$. Una biopsia positiva sin progresión clínica se consideró recurrencia local.

\section{Estadística}

El tiempo a la progresión fue calculado utilizando un modelo paramétrico de supervivencia Weibull Random Disturbance. El grupo de riesgo fue utilizado como variable independiente en el modelo. EL modelo se usó para predecir la probabilidad de progresión en el tiempo para cada uno de los grupos. La diferencia en progresión entre los grupos se testó utilizando análisis de los efectos tipo III, Chi cuadrado, el test del modelo descrito ${ }^{10}$.

\section{RESULTADOS}

Se presentan los resultados de aquellos pacientes que han recibido CSA como tratamiento primario. Las características de los pacientes según los grupos de riesgo se muestran en la Tabla 1. En el grupo de alto riesgo cuatro pacientes recibieron una inyección de depósito de análogos LH RH con la intención de disminuir el tamaño de la próstata previo a la CSA. Fueron excluidos del análisis aquellos pacientes que habían recibido antiandrógenos o análogos LHRH como tratamiento del cáncer de próstata. 
Tabla 1. Características de los pacientes previas a la ablación crioterapia prostática

\begin{tabular}{|c|c|c|c|c|}
\hline & $\begin{array}{l}\text { Bajo Riesgo } \\
\text { Media o Número } \\
\text { absoluto (rango o } \\
\text { \% dentro del grupo) }\end{array}$ & $\begin{array}{l}\text { Riesgo Intermedio } \\
\text { Media o Número } \\
\text { absoluto (rango o } \\
\% \text { dentro del grupo) }\end{array}$ & $\begin{array}{l}\text { Alto Riesgo } \\
\text { Media o Número } \\
\text { absoluto (rango o } \\
\text { \% dentro del grupo) }\end{array}$ & $\begin{array}{l}\text { Global } \\
\text { Media o Número } \\
\text { absoluto (rango o } \\
\text { \% dentro del grupo) }\end{array}$ \\
\hline No. De pacientes & 27 & 37 & 26 & 90 \\
\hline Edad (años) & $63,8(55-73)$ & $65,5(53-75)$ & $66,1(56-76)$ & $62,2(53-76)$ \\
\hline Volumen Prostatico(ml) & $32,6(19,9-70,7)$ & $33,7(14-56,3)$ & $36,4(18,3-70,4)$ & $32,7(14-70,7)$ \\
\hline $\begin{array}{l}\text { Estadios Enfermedad } \\
\text { T1 } \\
\text { T2a } \\
\text { T2b } \\
\text { T2c }\end{array}$ & $\begin{array}{c}16(59 \%) \\
11(41 \%) \\
0 \\
0\end{array}$ & $\begin{array}{l}11(30 \%) \\
10(27 \%) \\
16(43 \%) \\
0\end{array}$ & $\begin{array}{c}10(38,5 \%) \\
0 \\
5(19 \%) \\
11(42,5 \%)\end{array}$ & $\begin{array}{l}37(41 \%) \\
21(23 \%) \\
21(23 \%) \\
11(13 \%)\end{array}$ \\
\hline NO & 0 & 4 & 12 & $16(18 \%)$ \\
\hline NX & 27 & 33 & 14 & $74(82 \%)$ \\
\hline MO & $9(33 \%)$ & 16 & 16 & $41(46 \%)$ \\
\hline MX & $18(67 \%)$ & 21 & 10 & 49 (54\%) \\
\hline PSA (ng/ml) & $5,83(0,5-9,8)$ & $10,5(3,9-16)$ & $17.3(6-50)$ & $10,4(0,5-50)$ \\
\hline $\begin{array}{l}\text { Gleason score } \\
\quad \leq 6 \\
7 \\
>7\end{array}$ & $\begin{array}{c}27(100 \%) \\
0 \\
0\end{array}$ & $\begin{array}{c}28(76 \%) \\
9(24 \%) \\
0\end{array}$ & $\begin{array}{c}9(35 \%) \\
11(42 \%) \\
6(23 \%)\end{array}$ & $\begin{array}{l}64(71 \%) \\
20(22 \%) \\
6(7 \%)\end{array}$ \\
\hline
\end{tabular}

PSA = Antígeno prostático específico

Actualmente, el periodo de seguimiento más largo es de 42 meses. La mediana de seguimiento es de 21 meses y un $39 \%$ de los pacientes ya han sido vistos en revisión después de 24 meses.

No se ha observado evidencia de progresión clínica en el grupo de pacientes de bajo riesgo o alto riesgo. Dos pacientes en el grupo intermedio tuvieron evidencia clínica de progresión. (Tabla 2).

\section{Niveles de PSA}

Grupo Bajo Riesgo (LR): Los detalles de la mediana de PSA durante el seguimiento se muestran en la Fig. 4. Dos pacientes estabilizaron el nivel de PSA en aproximadamente $1 \mathrm{ng} / \mathrm{ml}$, lo que conllevó a un ligero incremento del valor de la mediana de PSA. Ningún paciente experimentó recidiva local o progresión del PSA.

Tabla 2. Detalle de los pacientes con evidencia de progresión de enfermedad después de ablación crioterápica prostática.

\begin{tabular}{|c|c|c|c|c|c|c|c|c|c|c|}
\hline $\begin{array}{l}\text { Edad } \\
\text { Paciente } \\
\text { (años) }\end{array}$ & $\begin{array}{l}\text { Gleason } \\
\text { score }\end{array}$ & TNM & $\begin{array}{l}\text { Grupo de } \\
\text { riesgo }\end{array}$ & $\begin{array}{c}\text { Tamaño } \\
\text { prostatico } \\
\text { (ml) }\end{array}$ & $\begin{array}{c}\text { PSA } \\
\text { inicial } \\
\text { (ng/ml) }\end{array}$ & $\begin{array}{c}\text { PSA } \\
\text { nadir } \\
\text { (ng/ml) }\end{array}$ & $\begin{array}{l}\text { Criterio de } \\
\text { progresión }\end{array}$ & Biopsia & $\begin{array}{c}\text { Tiempo a } \\
\text { evidencia de } \\
\text { progresión }\end{array}$ & $\begin{array}{l}\text { Tratamiento } \\
\text { secundario }\end{array}$ \\
\hline 72 & 6 & T1CNXMX & Intermed. & 44,3 & 10 & 4,2 & Incremento de PSA & Negativa & 3 meses & aLHRH \\
\hline 62 & 6 & T2aNXMX & Intermed. & $46, .5$ & 11 & 0.2 & Incremento de PSA & Negativa & 12 meses & Repetir CSA \\
\hline 68 & 7 & T2bNXMX & Intermed. & 20 & 12 & 0,5 & Incremento de PSA & Positiva & 12 meses & Repetir CSA \\
\hline 60 & 7 & T2aNOM0 & Intermed. & 47,4 & 15 & 4,2 & Incremento de PSA & Positiva & 3 meses & Repetir CSA \\
\hline 67 & 7 & T2NXMX & Intermed. & 41 & 5 & 0,07 & Metastasis Oseas & Negativa & 18 meses & aLHRH \\
\hline 66 & $\begin{array}{c}7 \text { (8 en la } \\
\text { biopsia }\end{array}$ & T2bNXMX & Alto & 27 & 7.3 & 1.9 & Incremento de PSA & Positiva & 3 meses & $\begin{array}{c}\text { RTE+ } \\
\text { bicalutamida }\end{array}$ \\
\hline 59 & $\begin{array}{c}7 \text { (9 en la } \\
\text { biopsia) }\end{array}$ & T2cNXMX & Alto & 24 & 11 & 0,6 & Incremento de PSA & Positiva & 6 meses & RTE+ aLHRH \\
\hline 73 & 8 & TlcNXMX & Alto & 48,7 & 26 & 1,0 & Incremento de PSA & Positiva & 12 meses & $\begin{array}{l}\text { Referido para } \\
\text { RTE+ aLHRH }\end{array}$ \\
\hline
\end{tabular}

PSA = antígeno prostático específico; aLHRH =análogo de LHRH; RTE=Radioterapia Externa 


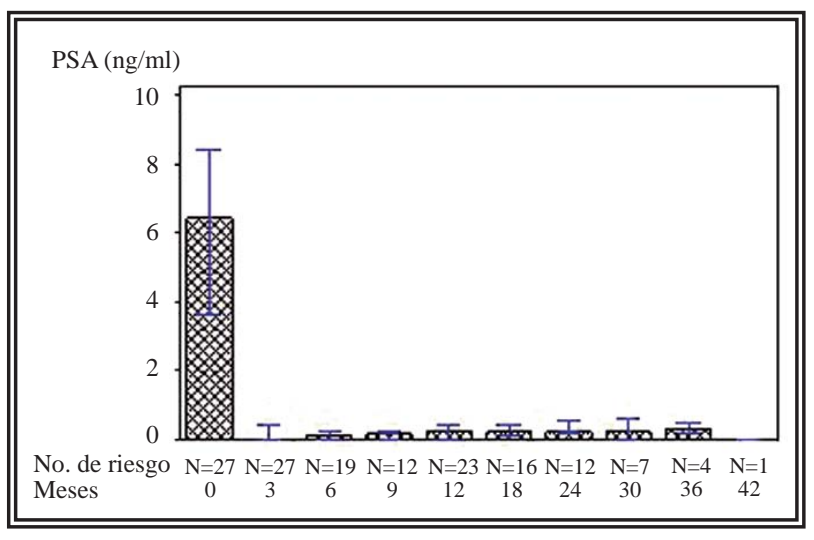

FIGURA 4. Mediana de PSA en los pacientes de bajo riesgo sometidos a crioterapia prostática. Las barras de error representan los valores del primer y el tercer cuartil.

Grupo Riesgo Intermedio: La mediana PSA y los valores de progresión del PSA durante el seguimiento se muestran en las Figuras 5 y 6. Los valores de PSA en dos pacientes causaron un incremento del valor de la mediana. Sus valores del PSA parecen haberse estabilizados entorno a 3,9 y $6 \mathrm{ng} / \mathrm{ml}$. Un paciente presentó crecimiento intravesical del lóbulo derecho después de una resección incompleta de la próstata por sintomatologia de hiperplasia benigna de próstata, previa a la CSA. Este lóbulo, probablemente no fue congelado completamente durante el procedimiento. Otro paciente tenía una larga historia de prostatitis crónica previa a la CSA. Cinco pacientes en el grupo de riesgo intermedio presentaron una progresión de PSA evidente (Tabla 2).

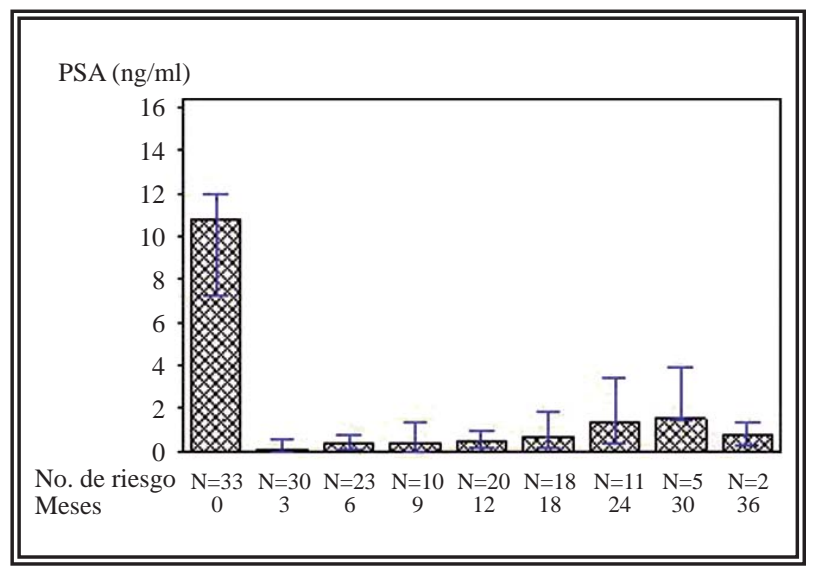

FIGURA 5. Mediana de PSA en los pacientes de riesgo intermedio sometidos a crioterapia prostática. Las barras de error representan los valores del primer y el tercer cuartil. Los datos de 4 pacientes con evidencia de progresión no son mostrados.

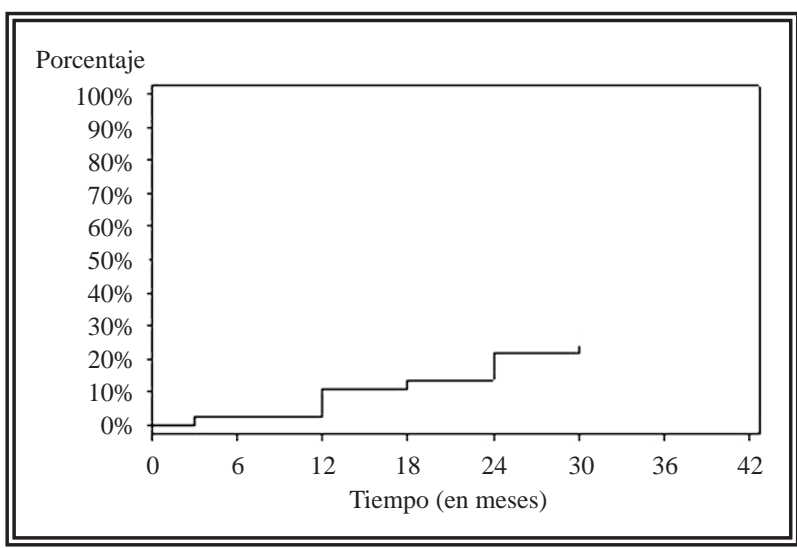

FIGURA 6. Curva de progresión clínica y de PSA en el grupo de riesgo intermedio sometidos a crioterapia prostática.

Grupo alto riesgo (HR): La mediana PSA y los valores de progresión del PSA durante el seguimiento se muestran en las Figuras 7 y 8 . Se informó de progresión de PSA en tres pacientes. Un paciente tenía un PSA preoperatorio de $21 \mathrm{ng} / \mathrm{ml}$. Su nadir fue de $2,5 \mathrm{ng} / \mathrm{ml}$ tres meses después, pero se estabilizó en valores entorno a $10-12 \mathrm{ng} / \mathrm{ml}$ a los 30 y 36 meses. Las biopsias fueron negativas. El segundo paciente tenía un PSA inicial de $28 \mathrm{ng} / \mathrm{ml}$ y un nadir de 1,2 $\mathrm{ng} / \mathrm{ml}$ y fue lentamente aumentando el valor del PSA. El tercer paciente tenía un PSA inicial de 13,7 $\mathrm{ng} / \mathrm{ml}$, alcanzó un nadir de $2,1 \mathrm{ng} / \mathrm{ml}$ y un valor del PSA 18 meses después de $5,1 \mathrm{ng} / \mathrm{ml}$.

\section{Modelo estadístico}

La probabilidad de progresión predichas después de 5 años para cada uno de los grupos fue de $1 \%$, 29\% y $32 \%$ para los grupos LR, IR y HR

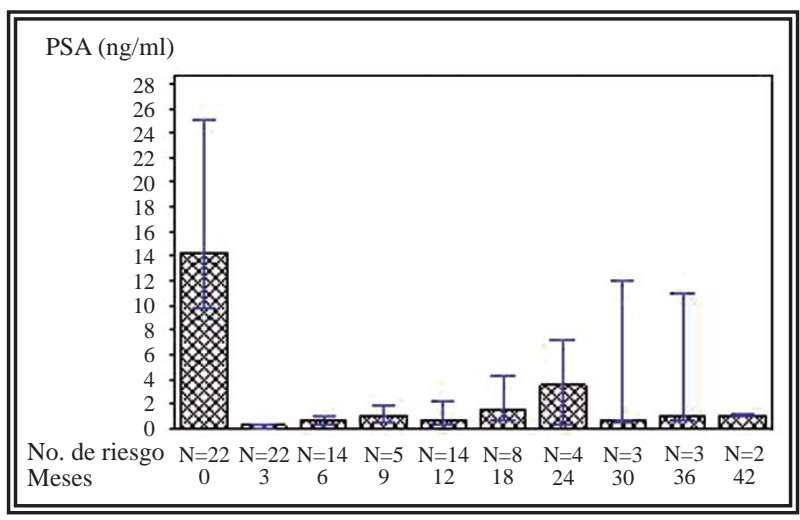

FIGURA 7. Mediana de PSA en el grupo de pacientes de alto riesgo sometidos a crioterapia prostática. Las barras de error representan los valores del primer y el tercer cuartil. Los datos de cuatro pacientes con evidencia de progresión no están mostrados. 


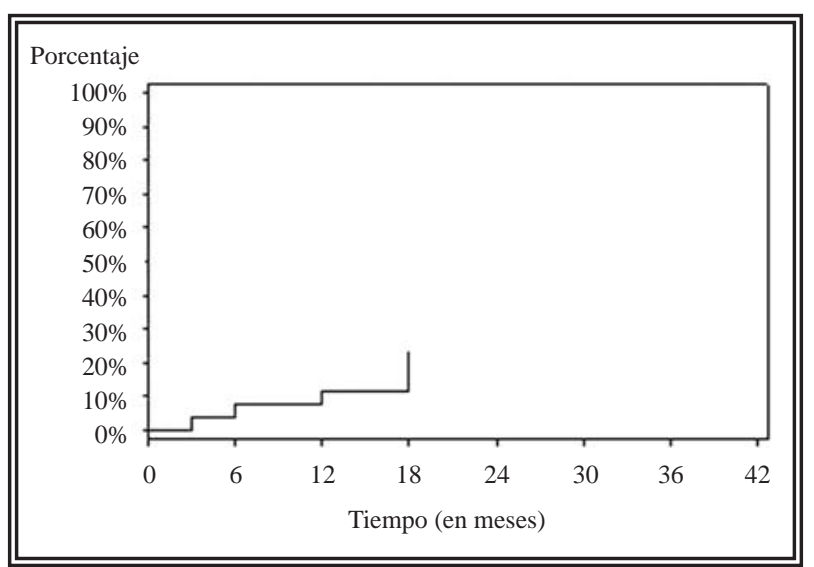

FIGURA 8. Curva de progresión clínica y de PSA en el grupo de alto riesgo sometidos a crioterapia prostática.

respectivamente (Fig. 9). No hubo diferencias estadísticamente significativas entre los grupos en términos de progresión ( $\mathrm{p}=0,982)$.

\section{Efectos secundarios}

Los detalles de los efectos secundarios se muestran en la Tabla 3. Los efectos secundarios más comunes durante el primer año después de CSA fueron la obstrucción del tracto urinario y la necesidad de resecar el tejido prostático necrosado o las calificaciones. El análisis histopatológico del tejido resecado no evidenció la presencia de cáncer de próstata. No se ha informado de casos de hematuria, incontinencia o fistula.

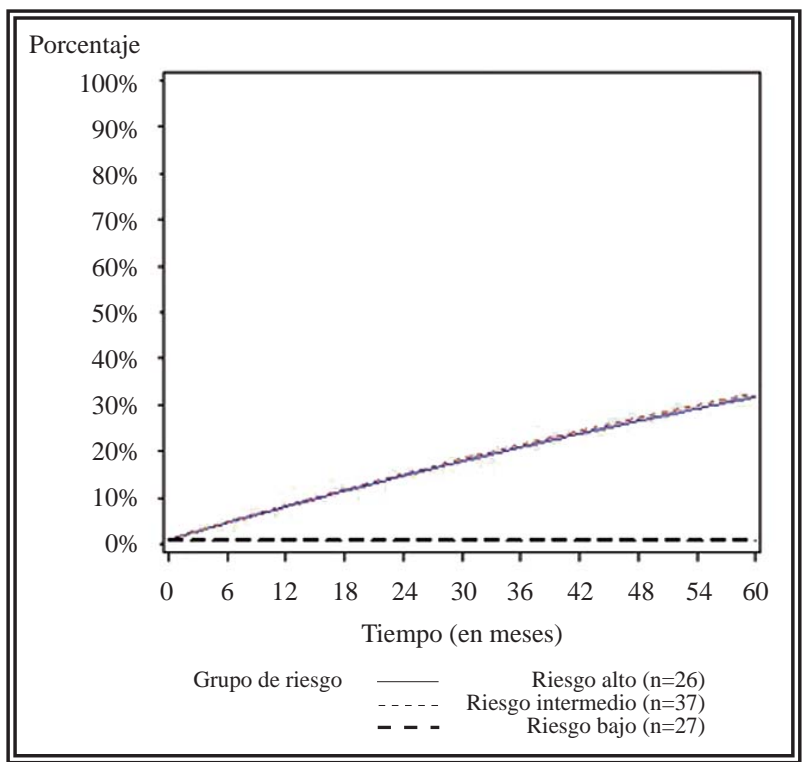

FIGURA 9. Modelo de Predicción de progresión Weibull Model por grupos de riesgo $(\mathrm{P}=0,982)$.

\section{Función Sexual}

Todos los pacientes en los grupos de riesgo bajo e intermedio que eran sexualmente actives antes del tratamiento lo eran en el último seguimiento, 37\% utilizaba algún tipo de ayuda. Un $86 \%$ de los pacientes de los grupos de riesgo bajo e intermedio, no refirieron alteraciones de su función sexual.

\section{DISCUSIÓN}

Un entrenamiento especial es necesario para la realización de CSA de la próstata y se observa una curva de aprendizaje fehaciente. Desde mi experiencia personal, los niveles de PSA cero post CSA fueron más frecuentes en mi segundo grupo de 30 pacientes que en el primero. Los factores que han contribuido a una mejoría de los resultados incluye la colocación de las agujas, alcanzar temperaturas menores en la próstata y el empleo más frecuente del procedimiento de empuje-retracción (Pull-back) para asegurar la adecuada cobertura de toda la glándula. La atención debe ser presentada a la anatomía de la próstata cuando el procedimiento de pull-back se realiza con el objetivo de no congelar estructuras innecesarias fuera de la próstata y con ello dañar los nervios. El empleo de catéter uretral mientras se realiza la colocación de las agujas ayuda a visualizar el cuello vesical y evitar una congelación innecesaria del trígono. A su vez, es muy importante evitar la compresión rectal por parte de 1 transductor ecográfico. El empleo de un Braquistandoff ha sido de mucha ayuda a la hora de levantar la próstata y hacerla encajar en la rejilla del ecografo, así como permitir la descompresión de la pared rectal conforme se expandía la bola de hielo. En adición, el empleo de un termosensor a nivel rectal, así como de dos agujas de calentamiento a nivel rectal, (Cytron-agujas) es muy importante en la protección de la pared rectal $^{11}$ (Fig. 3).

En un artículo reciente, discutíamos el criterio de progresión bioquímica tras tratamiento con CSA de $3^{\text {a }}$ generación ${ }^{8}$. Cuando se utiliza un catéter de calentamiento, algunas de las células prostáticas cerca de la uretra sobreviven y pueden producir PSA después. Las vesículas seminales tampoco son destruídas por la congelación. Sin embargo, si las células que sobreviven son 
Tabla 3. Efectos secundarios informados en 90 pacientes tratados con crioterapia prostática.

\begin{tabular}{|c|c|c|c|c|c|}
\hline & $\begin{array}{l}\text { Sangrado } \\
\text { N (\%) }\end{array}$ & $\begin{array}{l}\text { Incontinencia } \\
\quad \mathbf{N}(\%)\end{array}$ & $\begin{array}{c}\text { Infección tracto } \\
\text { urinario } \\
\mathrm{N}(\%)\end{array}$ & $\begin{array}{l}\text { Otra durante } \\
\text { primer año } \\
\text { N (\%) }\end{array}$ & $\begin{array}{c}\text { Otra después } \\
\text { del primer año } \\
\text { N (\%) }\end{array}$ \\
\hline $\begin{array}{l}\text { Grupo bajo riesgo } \\
(\mathrm{n}=27)\end{array}$ & 0 & 0 & 1 cistitis & $\begin{array}{c}1 \text { RTU-P } \\
1 \text { cistolitotricia }\end{array}$ & $\begin{array}{c}1 \text { ITU-P } \\
1 \text { cistolitotricia }\end{array}$ \\
\hline $\begin{array}{l}\text { Grupo riesgo Intermedio } \\
(\mathrm{n}=37)\end{array}$ & 0 & 0 & 0 & $\begin{array}{c}1 \text { RTU-P } \\
1 \text { CI durante } \\
\text { varias semanas }\end{array}$ & 1 RTU-P \\
\hline $\begin{array}{l}\text { Grupo alto riesgo } \\
(\mathrm{n}=26)\end{array}$ & 0 & 0 & 2 epididimitis & 0 & 0 \\
\hline Total & $0(0 \%)$ & $0(0 \%)$ & $3(3.3 \%)$ & $4(4.4 \%)$ & $3(3.3 \%)$ \\
\hline
\end{tabular}

RTU-P =Resección transuretral de próstata; ITU-P =Incisión transuretral de la próstata; CI = Cateterización Intermitente

benignas, el nivel de PSA debería estabilizarse. De hecho, este fenómeno lo hemos observado en algunos de nuestros pacientes. En un paciente del grupo de alto riesgo el PSA aumentó hasta 12 $\mathrm{ng} / \mathrm{ml}$ a los 6 meses y permaneció estable durante 3 años con biopsias negativas. Los criterios de la ASTRO recientemente desarrollados parece que se ajustan bien a lo que se considera un aceptable desarrollo del PSA después del nadir en pacientes tratados con $\mathrm{CSA}^{9}$. Sin embargo, uno debe tener en mente, que mientras para diagnosticar un cáncer de próstata puede ser suficiente con seis biopsias, para excluirlo es necesario realizar más biopsias. Seguimientos más largos son necesarios y nuestros pacientes serán seguidos con ese objetivo.

En publicaciones previas de crioterapia como tratamiento primario, un número significativo de pacientes estaban recibiendo o habían recibido algún tipo de tratamiento hormonal durante un periodo de tiempo considerable, lo cual podría interferir en los resultados ${ }^{12,13}$. Bahn ${ }^{12} \mathrm{y}$ Zisman ${ }^{13}$ presentaron unos excelentes resultados a largo plazo, pero un $91 \%$ y alrededor de un $40 \%$ de los pacientes habían recibido tratamiento hormonal durante un periodo de un año previo a la crioterapia. Con un periodo corto de observación esto puede tener un efecto significativo en el desarrollo de enfermedad. Nuestra serie puede ser considerada como constituida por pacientes sin tratamiento hormonal.

La cuestión clave es cuales son los histogramas de PSA y las curvas de progresión que veremos cuando todos los pacientes hayan completado cinco años de seguimiento. Los datos más prometedores en nuestro estudio son los vistos en los pacientes de bajo riesgo. Hay un pequeño incremento en el valor de la mediana de PSA en el grupo de bajo riesgo, pero la mayoría de los pacientes parece que alcanzan un valor estable del nivel de PSA. De acuerdo con el conocimiento de la EAU, una media del 33\% de los pacientes en el grupo de bajo riesgo experimentarán una recurrencia de PSA cinco años después de Prostatectomía Radical(PR) ${ }^{14}$. Uno debe ser muy cuidadoso a la hora de extrapolar nuestros resultados de 2 a 5 años. A su vez, es importante estar avisado de los distintos criterios de progresión de enfermedad después de PR y $\mathrm{CSA}^{8,9}$. Para el grupo de riesgo intermedio, había más pacientes con PSA in crescendo indicando que se verán más recurrencias o metástasis en los próximos años. Sin embargo, la probabilidad estimada de progresión después de 5 años es mucho menor del $50 \%$ de las recurrencias de PSA esperadas por la EAU. Es probable que nuestros resultados de progresión puedan alcanzar este nivel en dos años. Para el grupo de alto riesgo, una tasa de recurrencia de PSA del $85 \%$ se puede esperar a cinco años después de PR, lo que destaca el mal pronóstico de este grupo ${ }^{14}$. En nuestra serie, si combinamos los pacientes de riesgo intermedio y alto de manera conjunta, los resultados de progresión parecen ser muy optimistas.

Previamente, un efecto favorable de la CSA comparada con la radioterapia externa y la braquiterapia en los pacientes de intermedio y alto riesgo ha sido demostrado por Long ${ }^{15}$. Witsch et al. ${ }^{16}$ recientemente, presentaron resultados excelentes del European long term results para este grupo de pacientes. 
Todos los pacientes con progresión en nuestra serie pertenecían a los grupos intermedio y alto riesgo. De hecho, la progresión de PSA podría ser predicha, en la mayoría de los pacientes, por el valor del PSA nadir alrededor de los 3 meses. Con una estrategia más agresiva en las biopsias de estos pacientes podríamos identificar y referir los pacientes para un tratamiento de rescate mediante CSA o radioterapia externa en un momento más precoz.

Desde que el Scandinavian Prostate Cancer Group (SPCG)-IV study ${ }^{1}$ fue iniciado, el número de pacientes sometidos a tratamiento radical por cáncer de próstata ha aumentado por cuatro en el norte de Europa ${ }^{17}$ se ha multiplicado por cuatro y la selección de pacientes también ha cambiado con nuevos pacientes presentando tumores en un estadio más precoz de la enfer$\operatorname{medad}^{18}$ de manera que no influenciarán las curvas de mortalidad durante los primeros diez años después del tratamiento. Actualmente, necesitamos tratar más de 19 pacientes para salvar la vida de uno durante un periodo de diez años ${ }^{19}$

Posiblemente, menos del $2 \%$ de los pacientes se beneficiarán del efecto de salvar vidas que estamos buscando para este periodo. Sin embargo, el $100 \%$ de los pacientes estarán en riesgo de sufrir los efectos secundarios del tratamiento. El perfil de efectos secundarios en nuestra serie parece muy favorable y sustenta el concepto de la crioterapia como tratamiento mínimamente invasivo.

La crioterapia está reconocida por la EAU como una verdadera opción terapéutica alternativa, en el tratamiento del cáncer de próstata ${ }^{3}$. Múltiples instituciones ofrecen la crioterapia únicamente a pacientes añosos debido a la carencia de resultados a largo plazo. Si los resultados de un seguimiento más largo confirman los resultados obtenidos a corto plazo en esta serie, la crioterapia podría ser ofrecida también a pacientes más jóvenes como tratamiento primario. Una perspectiva atractiva de la crioterapia es la posibilidad de su utilización como tratamiento focal en pacientes con tumores diagnosticados en un estadio muy precoz. Además, ofrecer la posibilidad de congelar un solo lóbulo podría ayudar a preservar la eyaculación ${ }^{20}$.

\section{Agradecimientos}

Quiero agradecer a mi protector el Profesor Schmuel Cytron, quien me enseñó como hacer la CSA de la próstata; Moti Minsof por todas las ayudas técnicas; mis colegas por enviarme pacientes y ayudarme con el control en las visitas; Gro Hetterud por la asistencia de secretaria; y a Hilde Ufs y Torill Jacobsen por asistirme durante la mayoría de los procedimientos.

\section{REFERENCIAS}

1. Holmberg L, Bill-Axelson A, Helgesen F, Salo JO, Folmerz $\mathrm{P}$, Häggman $\mathrm{M}$, et al. A randomized trial comparing radical prostatectomy with watchful waiting in early prostate cancer. N Engl J Med. 2002;347:(11):781-789.

2. Steineck G, Helgesen F, Adolfsson J, Dickman PW, Johansson JE, Norlén BJ, et al. Quality of life after radical prostatectomy or watchful waiting. N Engl J Med. 2002; 347(11):790-796.

3. Heidenreich A, Aus G, Abbou CC, et al. EAU Guidelines on prostate cancer. In: European Association of Urology. Guidelines 2007 (ISBN-13:978-90-70244-59-0)

4. Bjerklund Johansen TE. Radical treatment of prostate cancer in Norway. Tidsskr Nor lægeforen. 2005;125:1658-60.

5. Haukaas SA, Halvorsen OJ, Daehlin L, Hostmark J, Akslen LA. Is preoperative serum prostate-specific antigen level significantly related to clinical recurrence after radical retropubic prostatectomy for localized prostate cancer? BJU Int. 2006;97(1):51-55.

6. Baust JG, Gage AA. The molecular basis of cryosurgery. BJU Int. 2005;95(9):1187-91.

7. Partin AW, Mangold LA, Lamm DM, Walsh PC, Epstein JI, Pearson JD. Contemporary update of prostate cancer staging nomograms (Partin Tables) for the new millennium. Urology. 2001;58(6):843-848.

8. Cytron S, Greene D, Witsch UKF, Nylund P, Bjerklund Johansen TE. Cryoablation of the prostate: Technical recommendations. Accepted for publication as part of EAU guidelines on prostate cancer. European Association of Urology. Guidelines 2007 (ISBN-13:978-90-70244-59-0).

9. Roach M, Hanks G, Thames H, Schellhammer P, Shipley WU, Sokol GH, et al. Defining biochemical failure following radiotherapy with or without hormonal therapy in men with clinically localized prostate cancer: recommendations of the RTOG-ASTRO phoenix consensus conference. Int J Radiation Oncology Biol Phys. 2006;65(4):965-974.

10. Lawless JE. Statistical Models and Methods for Lifetime Data. John Wiley \& Sons Inc: New York; 1982.

11. Cytron S, Paz A, Kravchick S, Shumalinski D, Moore J. Active rectal wall protection using direct transperineal cryo-needles for histologically proven prostate adenocarcinomas. Eur Urol. 2003;44(3):315-20; discussion 320-321.

12. Bahn DK, Lee F, Badalament R, Kumar A, Greski J, Chernick M. Targeted cryoablation of the prostate: 7-year outcomes in the primary treatment of prostate cancer. Urology. 2002;60(2 Suppl 1):3-11.

13. Zisman A, Pantuck AJ, Cohen JK, Belldegrun AS. Prostate cryoablation using direct transperineal placement of ultrathin probes through a 17-gauge brachytherapy template-technique and preliminary results. Urology. 2001;58 (6):988-993.

14. Abrahamsson PA. Treatment options for rising PSA after radical prostatectomy. European Urology Today. 2007;19 (1):2-3 
15. Long JP, Bahn D, Lee F, Shinohara K, Chinn DO, Macaluso JN. Five year retrospective, multi-institutional pooled analysis of cancer related outcomes after cryosurgical ablation of the prostate. Urology. 2001;57(3):518-523.

16. Witsch U, Dillenburg W, Hellwig W Skraipas K, Sucke C, Beht E. Cryoablation of prostate cancer using 17Gauge cryoneedles technology, 5 year European experience. Eur Urol. 2007;6(suppl 2):201, abstract 713.

17. Cooperberg MR, Park S, Carroll PR. Prostate cancer 2004: insights from national disease registries. 2004;18(10):12391247; discussion 1248-1250, 1256-1258.

18. de Vries SH, Postma R, Raaijmakers R, Roemeling S, Otto $\mathrm{S}$, de Koning HJ, et al. Overall and disease-specific survival of patients with screen-detected prostate cancer in the European randomized study of screening for prostate cancer, section Rotterdam. Eur Urol. 2007;51(2):366-374.
19. Bill-Axelson A, Holmberg L, Ruutu M, Häggman M, Andersson SO, Bratell S, et al. Radical prostatectomy versus watchful waiting in early prostate cancer. N Engl J Med. 2005;352(19): 1977-1984.

20. Onik G, Narayan P, Vaughan D, Dineen M, Brunelle R. Focal "nerve-sparing" cryosurgery for treatment of primary prostate cancer: a new approach to preserving potency. Urology 2002;60(1):109-114.

Correspondencia autor: Dr. T.E. Bjerklund Johansen Sección de Urología. Telemark Hospital. Prosgrunn, Norway Tel.: (47) 35004700

E-mail autor: tebi@sthf.no

Información artículo: Original 